\title{
Article
}

\section{Layer-by-layer assembled microgels can combine conflicting properties: Switchable stiffness and wettability without affecting permeability.}

Eliana Maza, Catalina von Bilderling, M. Lorena Cortez, Gisela Díaz, Micaela

Bianchi, Lía Isabel Pietrasanta, Juan Martín Giussi, and Omar Azzaroni

Langmuir, Just Accepted Manuscript • DOI: 10.1021/acs.langmuir.8b00047 • Publication Date (Web): 26 Feb 2018

Downloaded from http://pubs.acs.org on February 26, 2018

\section{Just Accepted}

"Just Accepted" manuscripts have been peer-reviewed and accepted for publication. They are posted online prior to technical editing, formatting for publication and author proofing. The American Chemical Society provides "Just Accepted" as a service to the research community to expedite the dissemination of scientific material as soon as possible after acceptance. "Just Accepted" manuscripts appear in full in PDF format accompanied by an HTML abstract. "Just Accepted" manuscripts have been fully peer reviewed, but should not be considered the official version of record. They are citable by the Digital Object Identifier (DOI®). "Just Accepted" is an optional service offered to authors. Therefore, the "Just Accepted" Web site may not include all articles that will be published in the journal. After a manuscript is technically edited and formatted, it will be removed from the "Just Accepted" Web site and published as an ASAP article. Note that technical editing may introduce minor changes to the manuscript text and/or graphics which could affect content, and all legal disclaimers and ethical guidelines that apply to the journal pertain. ACS cannot be held responsible for errors or consequences arising from the use of information contained in these "Just Accepted" manuscripts. 


\title{
Layer-by-layer assembled microgels can combine conflicting properties: Switchable stiffness and wettability without affecting permeability.
}

\author{
Eliana Maza, ${ }^{a}$ Catalina von Bilderling, ${ }^{\mathrm{a}, \mathrm{b}}$ M. Lorena Cortez, ${ }^{\mathrm{a}}$ Gisela Díaz, ${ }^{\mathrm{a}}$ Micaela Bianchi, ${ }^{\mathrm{d}}$ Lía I. \\ Pietrasanta, ${ }^{\text {,cc,d }}$ Juan M. Giussi, ${ }^{\text {a }}$ and Omar Azzaroni ${ }^{\text {a* }}$ \\ ${ }^{a}$ Instituto de Investigaciones Fisicoquímicas teóricas y Aplicadas (INIFTA). Consejo Nacional de \\ Investigaciones Científicas y Técnicas (CONICET)-Universidad Nacional de La Plata (UNLP). \\ Diagonal 113 y 64 S/N, 1900 La Plata, Bs. As., Argentina. \\ ${ }^{b}$ Departamento de Física, Facultad de Ciencias Exactas y Naturales, Universidad de Buenos Aires, \\ C1428EHA Buenos Aires, Argentina. \\ ${ }^{c}$ Instituto de Física de Buenos Aires (IFIBA, UBA-CONICET), Facultad de Ciencias Exactas y \\ Naturales, Universidad de Buenos Aires, C1428EHA Buenos Aires, Argentina. \\ ${ }^{d}$ Centro de Microscopías Avanzadas, Facultad de Ciencias Exactas y Naturales, Universidad de \\ Buenos Aires, C1428EHA Buenos Aires, Argentina
}

\begin{abstract}
Responsive interfacial architectures of practical interest commonly require the combination of conflicting properties in terms of their demand upon material structure. Switchable stiffness, wettability and permeability, key features for tissue engineering applications, are in fact known to be exclusively interdependent. Here we present a nanoarchitectonic approach that decouples these divergent properties by the use thermo-responsive microgels as building blocks for the construction of three-dimensional arrays of interconnected pores. Layer-by-layer assembled poly(N-isopropylacrylamide-co-methacrylic acid) (PNIPAM-co-PMAA) microgel films were found to exhibit an increase in hydrophobicity, stiffness and adhesion properties upon switching the temperature from below to above the lower critical solution temperature, while the permeability of redox probes through the film remained unchanged. Our findings indicate that the switch in hydrophilicity and nanomechanical properties undergone by the microgels does not compromise the porosity of the film, thus allowing the free diffusion of redox probes through the polymer-free volume of the submicrometer pores. This novel approach for decoupling conflicting properties provides a strategic route for creating tailorable scaffolds with unforeseen functionalities.
\end{abstract}

\section{Introduction}

The possibility of creating interfacial architectures displaying conflicting and seemingly mutually exclusive properties to achieve multiple functionalities is one of the grand challenges for materials science in the 21 st century. ${ }^{1-3}$ One broad challenge is to combine switchable stiffness and wettability

\footnotetext{
*e-mail: catalinavb@inifta.unlp.edu.ar azzaroni@inifta.unlp.edu.ar
} 
with high permeation properties from aqueous solutions. ${ }^{4,5}$ The strong interest in combining this set of properties arises from their potential applications in biomedical research and, in particular, in tissue engineering as well as cell adhesion control and manipulation. ${ }^{6,7}$ As is well known, the design and fabrication of tailorable scaffolds has been a key to the success of tissue engineering. ${ }^{8-10}$ This notion is even more relevant if we consider that the integration of thermoresponsive units in the scaffold and/or platform facilitates remote control over cell-surface interactions by switching the environmental temperature. This concept was pioneered by Okano an co-workers ${ }^{11}$ employing poly $(N$ isopropylacrylamide) (PNIPAM) as thermoresponsive units and led to the development of the so-called temperature-responsive cell culture surfaces. PNIPAM undergoes a solubility transition at the lower critical solution temperature (LCST) of $32^{\circ} \mathrm{C}$. The PNIPAM surface has the extraordinary property that at $32{ }^{\circ} \mathrm{C}$, the surface becomes hydrophobic allowing for cell attachment and proliferation. Then, upon decreasing temperature below the LCST, the surface becomes hydrophilic creating a hydration layer such that the cell sheet with deposited extracellular matrix detaches spontaneously. Since then, diverse groups exploited the temperature-dependent interactions between proteins and PNIPAM-coated surfaces to create substrates that reversibly capture proteins or cells. However, it is now widely accepted that these types of substrates should exhibit not only switchable wettability and stiffness to control cell adhesion, but they should also display high permeability in aqueous solution in order to maximize nutrient delivery and metabolite removal. This poses conflicting property requirements in multiple physico-chemical domains, i.e.: hydrophobicity and stiffness versus aqueous permeability.

In the case of PNIPAM-based polymer films, it has been demonstrated that the thermoactuation of the polymer chains strongly compromise the permeation properties. As already observed in other branches of materials science, ${ }^{12}$ stiffness and permeability are competing materials properties. In 2005, Jaber and Schlenoff $^{13}$ studied the permeation properties of thermally responsive polyelectrolyte multilayers constituted of charged PNIPAM copolymers. They observed that the transport of an electrochemically active probe molecule, potassium ferricyanide, through the thin film was substantially suppressed as temperature increased above the LCST. The same group studied the correlation between permeation properties and stiffness in polyelectrolyte multilayers corroborating the conflicting nature of these two properties. They observed that an increase in modulus was unambiguously correlated with a decrease in the permeability to iodide ion. ${ }^{14}$ The same correlation was also observed in PNIPAM brushes ${ }^{15}$ and hydrogels $^{16}$; a thermo-induced increase in stiffness or hydrophobicity was associated with a concomitant decrease in permeability. In principle, the permeability and mechanical properties of water-swollen systems such as polyelectrolyte multilayers, polymer brushes or hydrogels are expected to be strongly correlated because softer, more hydrated materials also tend to be more permeable. These observations suggest that permeability properties in polymer thin films cannot be decoupled from their nanomechanical or even wetting properties. This has strong implications for consistent application of switchable, thermoresponsive polymer films in cell and tissue engineering. As recently noted by Volodkin and coworkers, ${ }^{17}$ there is a strong need to design polymer films possessing reservoir and permeation capacity, and cell adhesive properties. Within this framework, the real challenge is to design polymer films with switchable nanomechanical, wetting and adhesion properties but keeping the reservoir and permeation properties of the films unaffected.

Most materials design problems of practical interest involve solutions with property/response sets that conflict in terms of their demand upon material structure at various scales. In recent years, computational modeling has demonstrated that microstructural composites represent a promising strategy to design and fabricate multifunctional materials combining conflicting properties. According to these computational studies, a multiphase microstructural configuration might facilitate the combination of properties from different building blocks without disrupting their own functions. ${ }^{18}$ With this in mind, we drew upon a "nanoarchitectonic approach"19-22 to develop switchable polymer films 
displaying conflicting properties, such as stiffness and permeability. The goal of our research was to integrate materials and length scales in a rational manner in order to decouple divergent functional requirements, thus far considered to be strongly correlated and exclusively interdependent functional attributes. To this end, we have exploited the submicrometer structure of microgels and the versatility of layer-by-layer assembly to build-up nanoarchitectured, opal-like thermoresponsive films that act as matrices containing arrays of three-dimensional interconnected pores. Layer-by-layer assembled poly(N-isopropylacrylamide-co-methacrylic acid) (PNIPAM-co-PMAA) microgel films exhibited an increase in hydrophobicity, stiffness and adhesion properties upon switching the temperature from below to above the LCST, but the permeability of redox probes through the film remained unaffected. Our results indicate that due the nanoarchitectured nature of the film, the switch in hydrophilicity and nanomechanical properties undergone by the microgels does not compromise the porosity of the film, thus permitting the free diffusion of redox probes through the polymer-free volume of the submicrometer pores. To the best of our knowledge, this is the first attempt to rationally combine conflicting properties in thin polymer films using a layer-by-layer assembly approach. We envision that our approach for decoupling divergent functional properties using hierarchically organized films would provide a facile route from creating interfacial architectures with unforeseen functional combinations.

\section{Results and Discussion}

The use of microgels as building blocks to construct polymeric films via layer-by-layer assembly was first reported by Lyon and co-workers. ${ }^{23}$ Since then, extensive research was conducted on the construction of responsive microgel films. ${ }^{24-29}$ One of the most appealing aspects of using thermoresponsive PNIPAM-co-PMAA microgels as polyanions in layer-by-layer assembled microgel films relies on the fact that we can create interfacial architectures with hierarchical microscale-tonanoscale structures that can actuate and change their properties upon varying the temperature. In the case of PNIPAM-co-PMAA microgels, they exhibit a well-defined LCST at $32{ }^{\circ} \mathrm{C}$ where the system undergoes a volume phase transition to a collapsed state with a concomitant release of solvent (water) from the interior of the microgel. The layer-by-layer construction of the hierarchically organized microgel films was accomplished following the protocol described by Decher and co-workers ${ }^{30,31}$ using PNIPAM-co-PMAA microgels and poly(diallyldimethylammonium chloride) (PDADMAC) as polyanions and polycations, respectively.

The build-up of PNIPAM-co-PMAA microgel/PDADMAC multilayer films (fig. 1) was monitored by microgravimetric measurements using a quartz crystal microbalance (QCM). Assembly of films of anionic PNIPAM-co-PMAA microgel and cationic PDADMAC was done by 15 min alternate adsorption from their respective solutions at $\mathrm{pH} 6.5$ (at room temperature). QCM monitoring of dry films showed a monotonic increase in frequency upon increasing the number of adsorption steps. The frequency shift is proportional to the mass of each adsorbed layer and indicates an increase of film mass on the QCM sensor with increasing number of deposition cycles (see Figure S1). 


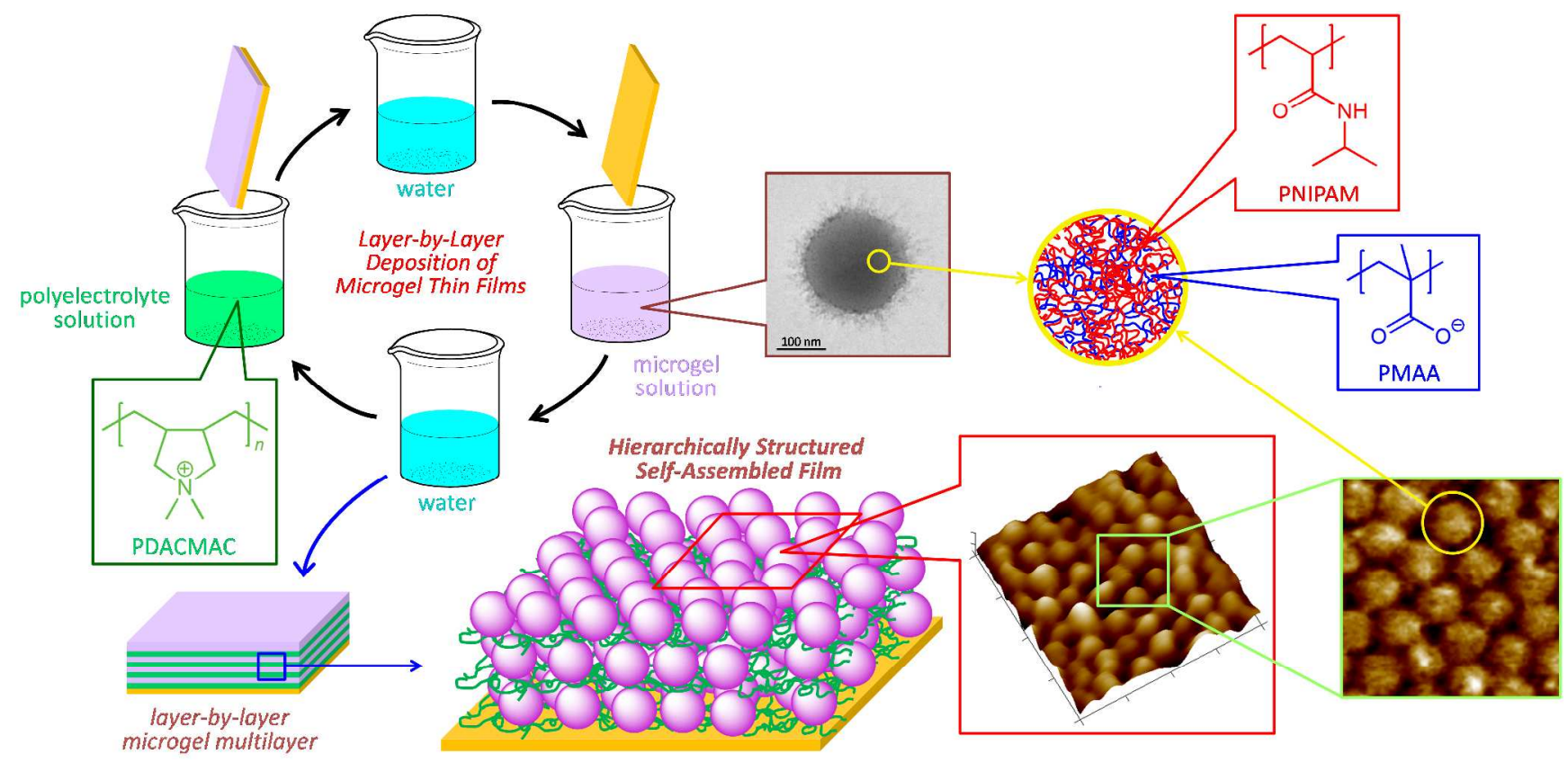

Figure 1. Schematic of the LbL deposition process using PDADMAC and PNIPAM-co-PMAA microgels as polycations and polyanions, respectively. PNIPAM-co-PMAA microgel samples were characterized by TEM imaging (scale bar: $100 \mathrm{~nm}$ ), and films were characterized by in-situ AFM imaging (height image $2 \times 2 \mu \mathrm{m}^{2}$ and phase image $1 \times 1 \mu \mathrm{m}^{2}$, color represents a total range of $100 \mathrm{~nm}$ in the height image). Note that the interfacial architecture depicted in the figure corresponds to a simplified representation of the film structure, in which anionic microgels are surrounded by polycation chains. However, partial diffusion of the polyelectrolytes into the microgels is also a plausible configuration that should be taken into account.

Once assembled, films were characterized by in-situ atomic force microscopy. Figure 1 shows an AFM image of a (PNIPAM-co-PMAA/PDADMAC)//PNIPAM-co-PMAA multilayered film. It can be observed that the layer-by-layer assembly process led to a film exhibiting close-packed microgel on the outermost layer.

Previous studies reported by von Klitzing and coworkers suggest that, in principle, microgels containing charged monomers cannot lead to the formation of close-packed films due to strong interparticle microgel-microgel repulsion. ${ }^{29,32}$ However, the same authors conclude that there are two electrostatic contributions that control the formation of the microgel monolayer: (i) electrostatic repulsion between charged microgels, and (ii) attractive electrostatic interactions between the substrate and the microgels. According to their results, when $\mathrm{pH}$ conditions range between 6 and 10, there is a trade-off between attractive and repulsive interactions. From the results, we can infer that the assembly of the microgel on the PDACMAC layer rather than microgel-microgel repulsion governs the formation of each layer. This could be ascribed to the presence of salt in the assembly solution that ultimately screens the repulsive electrostatic interactions between charged microgels.

Once confirmed the structure of the film at room temperature (below LCST) we proceeded to study the morphological changes taking place in the sample upon increasing temperature above the LCST. We should note that, thus far, in-situ AFM imaging of morphological transformations as well as changes in adhesion and nanomechanical properties of LbL-assembled microgel multilayers in response to temperature have not been reported in the literature. 

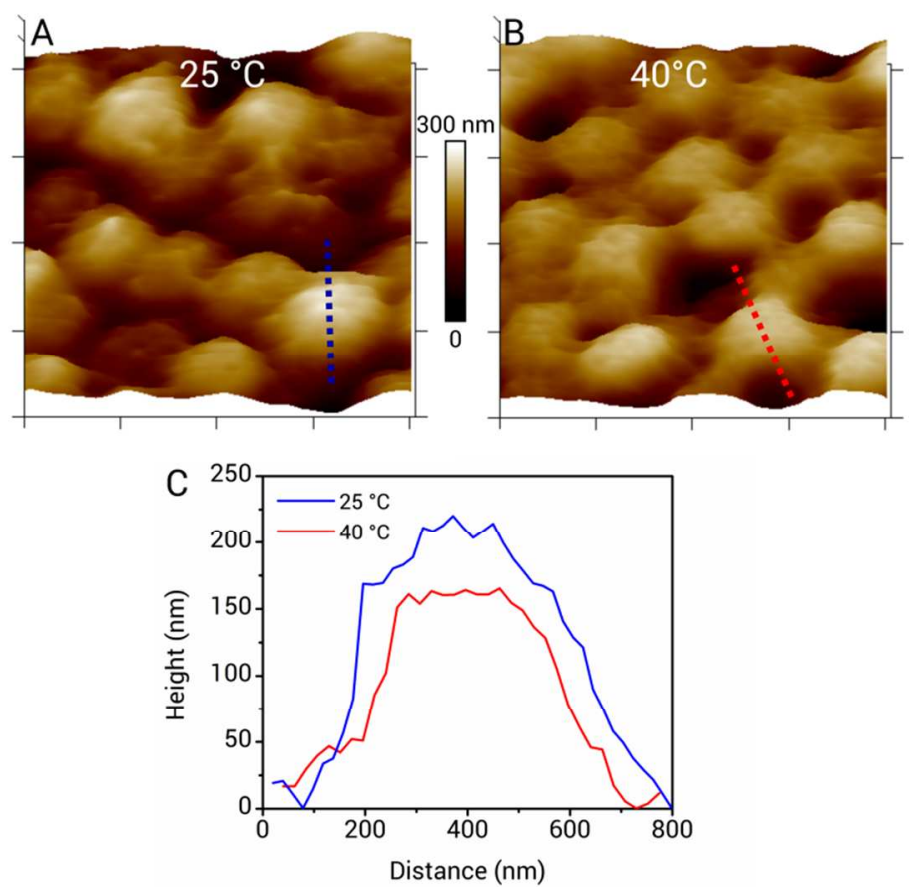

Figure 2. Topography AFM images of the (PNIPAM-co-PMAA/PDADMAC) 9 /PNIPAM-co-PMAA multilayered film at $25(\mathbf{A})$ and $40{ }^{\circ} \mathrm{C}(\mathbf{B})$. Images are presented as surface plots to emphasize features. Scan size $2 \times 2 \mu^{2}$. (C) From each image, a cross-sectional analysis of individual microgel particles was performed as the shown from lines. No significant changes were observed, neither in the surface morphology nor at the profiles mean FWHM diameters which resulted $(390 \pm 50) \mathrm{nm}$ and $(370 \pm 40) \mathrm{nm}$ at 25 and $40{ }^{\circ} \mathrm{C}$ respectively (for 10 profiles at each condition, standard deviations as errors).

We should bear in mind that precise topographic measurement of the outermost microgel layer is not trivial due to the softness of the imaged sample. ${ }^{33}$ However, we were able to reliably image the surface by using the high-frequency force tapping mode (Bruker's Quantitative Nanomechanics, QNM), for which the AFM tip briefly interacts with the sample surface thus minimizing the possible deformation of the microgels during the imaging process. ${ }^{34}$ Figure 2 depicts the morphology of the (PNIPAM-coPMAA/PDADMAC) $)_{9} /$ PNIPAM-co-PMAA multilayered film as obtained from in-situ AFM characterization performed at 25 and $40{ }^{\circ} \mathrm{C}$. It is observed that no significant morphological changes take place in the sample upon increasing temperature above LCST. Indeed, by evaluating AFM height profiles of individual microgel particles at the outermost layer of the film (Figure 2C), we obtained mean FWHM diameters of $(390 \pm 50) \mathrm{nm}$ and $(370 \pm 40) \mathrm{nm}$ for 25 and $40{ }^{\circ} \mathrm{C}$ respectively. The absence of major morphological changes across the LCST also implies that the film does not undergo significant changes in the dimensions of the submicron interparticle cavities (as observed in the AFM images). Or, in other words, the temperature increase does not significantly affect the volume of the macroporous space generated by the three-dimensional interconnected cavities. Dynamic light scattering measurements of these microgels dispersed in solution confirmed that the hydrodynamic diameter varies from 1400 to $200 \mathrm{~nm}$ in the same conditions in which they were LbL-assembled, i.e.: microgels in solution deswell by a factor of $1 / 7$ in diameter. ${ }^{35}$ Differences observed in deswelling between microgels dispersed in solution and integrated in LbL assemblies are likely attributable to the interaction with the underlying polyelectrolyte layer. For instance, Höfl et al. ${ }^{28}$ observed that poly $(\mathrm{N}-$ isopropylacrylamide)-co-vinylacetic acid microgel particles adsorbed onto a solid substrate display a similar volume phase transition as their dispered counterparts. However, surprisingly, their swelling 
capacity was reduced by one order of magnitude compared to value of microgels in solution. Similarly, Schmidt et al. ${ }^{36}$ reported that negatively charged PNIPAM microgels adsorbed on positively charged polyethyleneimine layers exhibit a reduced swelling/deswelling capacity.

From these results, we infer that the microgel integration and confinement into the multilayer assembly - a scenario in which these building blocks are surrounded by polyelectrolyte layers - exerts a marked influence on the shape and dimensional actuation of the microgels, which in turn defines not only the texture but also the hierarchical micro/nanotopography of the interfacial architecture.

Then, we proceeded to study the influence of the thermal conditions on the nanomechanical and adhesion properties of the LbL-assembled microgel multilayers. Probing of the surface mechanical properties with nanometer-scale resolution was accomplished via atomic force microscopy characterization. Despite some technical issues associated with this AFM probing (e.g.: nanoscale contact area or high local pressure), a number of successful applications of this experimental approach have been demonstrated to date. ${ }^{37-42}$

Figure 3A depicts typical AFM force-distance curves (FDC) performed on the microgel multilayer under different temperature conditions, i.e.: below and above LCST. We defined an upper force threshold of $2 \mathrm{nN}$, which led to deformations on the order of $100 \mathrm{~nm}$. High agreement on subsequent measurements on the same spot indicated that the surface was not plastically deformed during nanomechanical characterization.
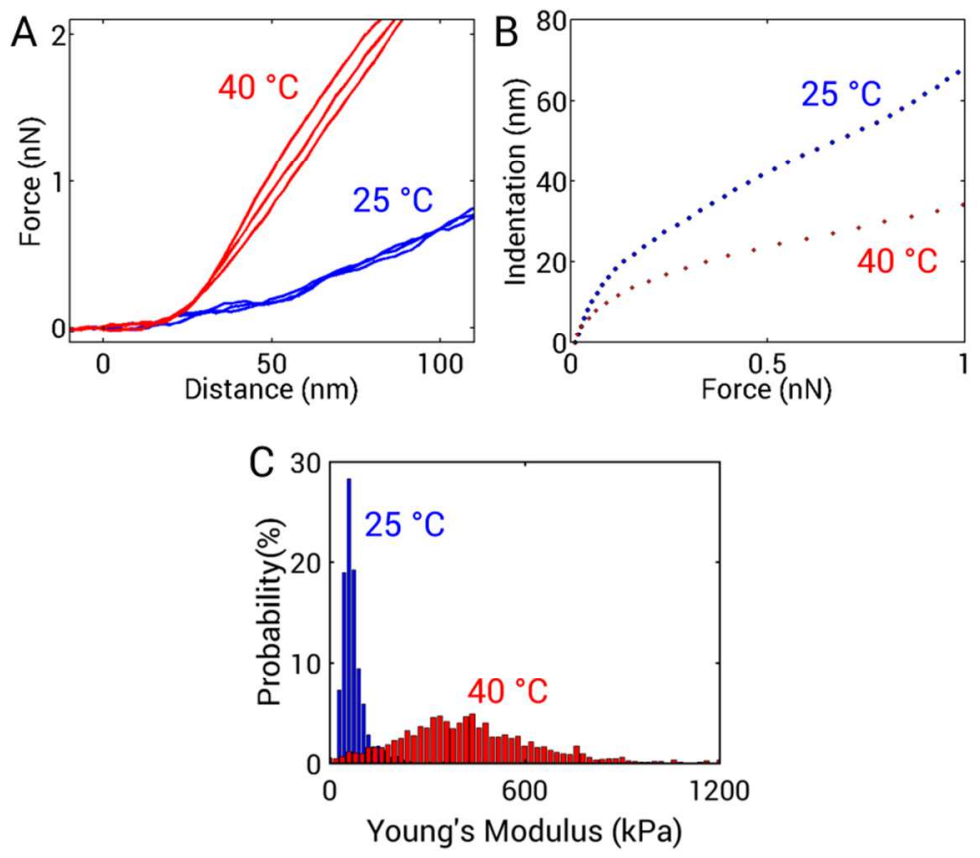

Figure 3. (A) Typical AFM loading force distance curves performed at $25{ }^{\circ} \mathrm{C}$ (blue) and $40{ }^{\circ} \mathrm{C}$ (red). (B) Plot of the indentation vs. the applied force (mean data from 20 FDC). (C) Young's modulus histograms obtained by the Sneddon's contact mechanics model (from $\sim 1500$ FDC). We obtained mean Young's modulus values of (75 \pm $41) \mathrm{kPa}$ for $25^{\circ} \mathrm{C}$ and $(450 \pm 210) \mathrm{kPa}$ for $40^{\circ} \mathrm{C}$ (standard deviations as errors).

Visual inspection of the FDC showed a clear difference on the mechanical response of the film for temperatures below and above the LCST. Conversion of the FDCs into load penetration curves further confirmed that a more compliant surface is associated with the $25{ }^{\circ} \mathrm{C}$ state, as indentation is almost twice as deep under a normal load of $1 \mathrm{nN}$ (Figure 3B). 
Elastic response of AFM force-distance data was analyzed by the Sneddon's contact mechanics model, assuming a parabolic tip and a plane surface contact. Knowledge of the force applied to the indenter as a function of the penetration during the loading experiment allows for the calculation of Young's modulus $E$ using a numerical form of the Sneddon's equation for AFM data, which has been successfully applied in previous reports ${ }^{39,43}$ (further technical details are described in the SI). An analysis of these data indicated a 6-fold increase of the modulus when increasing the temperature from 25 to $40{ }^{\circ} \mathrm{C}(75 \pm 41 \mathrm{kPa}$ and $450 \pm 210 \mathrm{kPa}$, respectively). The variation in modulus observed in microgel multilayer is comparable to a previous study on thermoreponsive microgel monolayers. ${ }^{36}$ This variation on nanomechanical properties is fully reversible (see Figs. S2 and S3 in the SI file). Furthermore, analyzing force curves from a linear profile along the section of a microgel within the opal-like structure, we observed that the elastic modulus increases at positions closer to the apex (see Figure 4 A-D). A similar observation was previously reported by Schmidt et al. in a set of experiments performed on microgel monolayers ${ }^{36}$. These results could be explained considering that in many cases the polymerization of PNIPAM microgels results in the formation of a highly crosslinked core and a shell with lower density. In our case, we have corroborated the existence of this structural feature in our PNIPAM-co-PMAA microgel samples by TEM imaging (see Fig. 1).

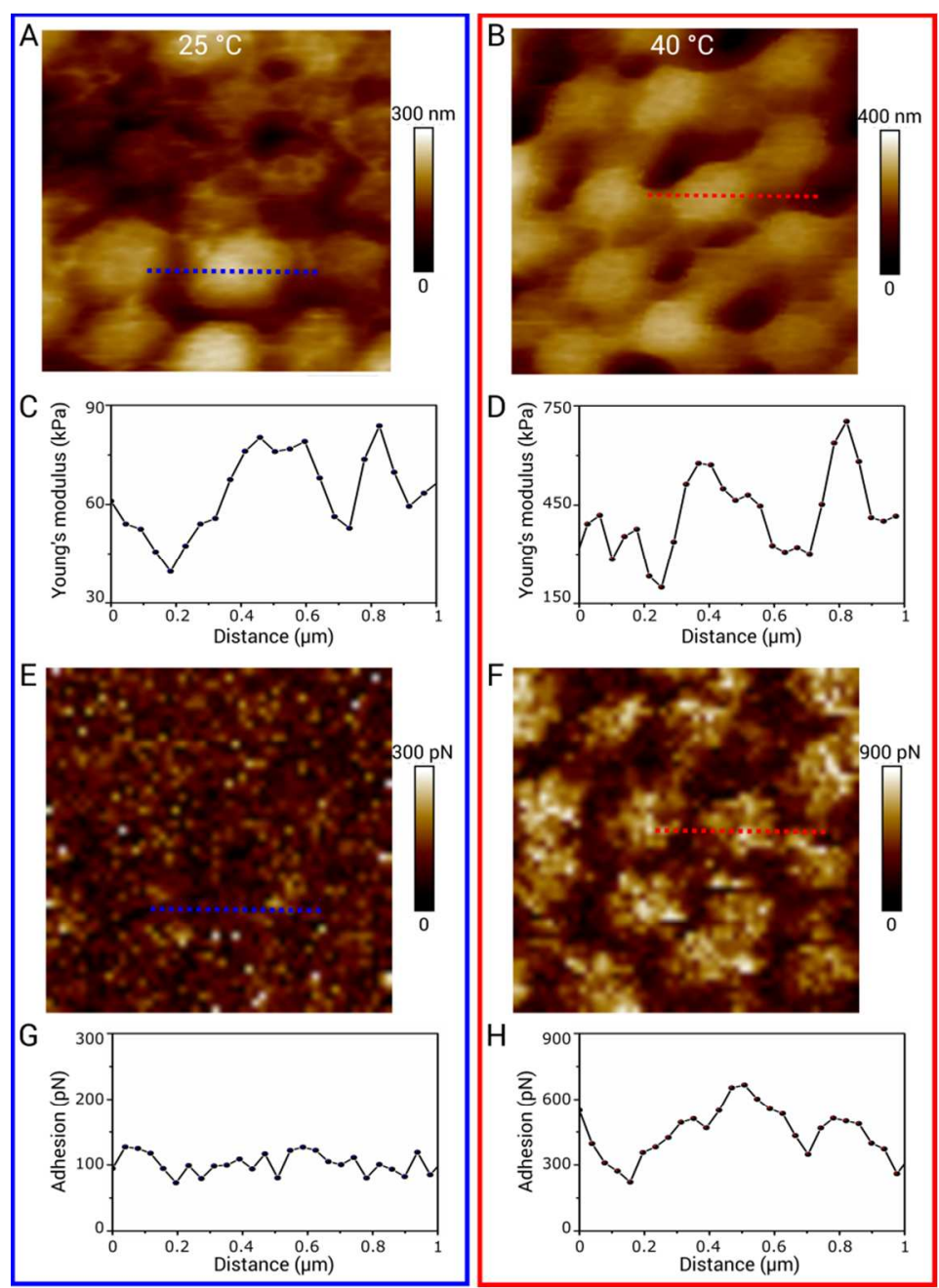

Figure 4. (A, B) $2 \times 2 \mu \mathrm{m}^{2}$ topography AFM images of the (PNIPAM-co-PMAA/PDADMAC) $)_{9} /$ PNIPAM-coPMAA multilayered film $25{ }^{\circ} \mathrm{C}(\mathrm{A})$ and $40{ }^{\circ} \mathrm{C}(\mathrm{B})$. (C, D) Elastic modulus profiles along a microgel cross- 
section (blue and red lines in A and B respectively). (E-H) $2 \times 2 \mu \mathrm{m}^{2}$ adhesion force maps of the imaged regions, and adhesion force cross-sections along the red and blue lines.

In a similar vein, we explored the adhesion response of the microgel multilayer by AFM nanomechanical mapping. QNM adhesion force maps at 25 and $40{ }^{\circ} \mathrm{C}$ showed a significant adhesion increase above the LCST, particularly on microgels surfaces, which was evidenced by the adhesion images contrast as well as in their cross-sections (Figure $4 \mathrm{E}-\mathrm{H}$ ).

In order to avoid possible artifacts from the fast rate nanomechanical mapping scans, we proceed to quantify the adhesion from single FDC taken at random locations of the multilayer surface. We evaluated the work required to pull of the AFM probe from the surface as a measure of the adhesion energy. Work of adhesion can be read from the AFM retraction curves as the area enclosed by the force curve and the $x$-axis (Figure $5 \mathrm{~A}$ ). We observed a gradual increase in the mean work of adhesion from $(0.53 \pm 0.15) \times 10^{-16} \mathrm{~J}$ to $(1.33 \pm 0.25) \times 10^{-16} \mathrm{~J}$ when temperature was increased from $25{ }^{\circ} \mathrm{C}$ to $40{ }^{\circ} \mathrm{C}$ respectively (Figure 5B). This behavior, observed in previous studies on PNIPAM microgel monolayers, ${ }^{44,45}$ is in close resemblance to the adhesion behavior of thermoresponsive polymer brushes $^{46-48}$ and can be ascribed to the fact that above the LCST the solvent is depleted from the macromolecular environment and the polymer chains/segments collapse. This process leads to large adhesion forces due to van der Waals interactions between the polymer layer and the AFM tip. On the other hand, below the LCST the microgel is swollen and the microgel-tip interaction is mainly governed by repulsive osmotic forces which in turn lead to a decrease in adhesion forces.

In addition, and as expected, the switching in the adhesion properties of the (PNIPAM-coPMAA/PDADMAC) 9 /PNIPAM-co-PMAA assembly upon increasing temperature from 25 to $40{ }^{\circ} \mathrm{C}$ was accompanied by an increase in the contact angle of the microgel assembly from 71 to $95^{\circ}$ (Figure 5 C-D), i.e.: an increase in hydrophobicity. Note that this wettability switch is fully reversible and multilayer assemblies exhibit an increase in hydrophilicity upon decreasing the working temperature below LCST (see Fig. S4 in the SI file).
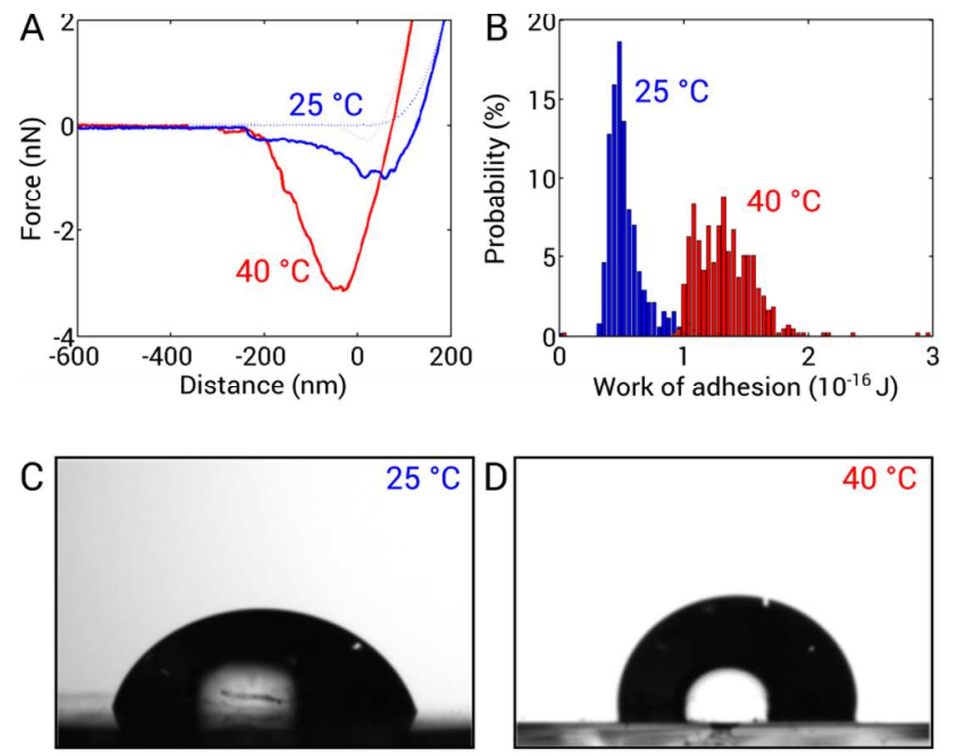

Figure 5. (A) Representative AFM unloading FDC performed at $25{ }^{\circ} \mathrm{C}$ (blue) and $40{ }^{\circ} \mathrm{C}$ (red). (B) Work of adhesion histograms, calculated for about $500 \mathrm{FDC}$ at each condition by evaluating the area enclosed by the retraction force curve and the $x$-axis. Mean values of $(0.53 \pm 0.15) \times 10^{-16} \mathrm{~J}$ and $(1.33 \pm 0.25) \times 10^{-16} \mathrm{~J}$ were 
obtained for $25^{\circ} \mathrm{C}$ and $40{ }^{\circ} \mathrm{C}$ respectively (standard deviations as errors). (C-D) Contact angle images taken at $25^{\circ} \mathrm{C}(\mathrm{C})$ and $40{ }^{\circ} \mathrm{C}(\mathrm{D})$.

So far, these results are similar to those previously reported in the literature using PNIPAM-based films. Next, we studied the transport properties of the multilayered microgel films under different temperature conditions. These studies were carried out using redox probes, $\mathrm{Fe}(\mathrm{CN})_{6}^{4-3-}$, that diffuse across the microgel multilayer grown on a conductive gold substrate. Electrochemical experiments were performed in $0.1 \mathrm{M} \mathrm{KCl}$ as supporting electrolyte. We should note that this saline concentration did not affect the structural integrity of the self-assembled film, as corroborated by AFM characterization (see Fig. S5 in the SI file). Figure 6 describes the voltammograms of (PNIPAM-coPMAA/PDADMAC) $)_{9} /$ PNIPAM-co-PMAA multilayers in contact with electrolyte solutions containing $\mathrm{Fe}(\mathrm{CN})_{6}{ }^{4-3-}$. We observed that - in stark contrast with the vast literature on PNIPAM films ${ }^{14-16}-\mathrm{a}$ temperature increase above the LCST does not promote changes in the permeation of the electroactive $\mathrm{Fe}(\mathrm{CN})_{6}^{4-/ 3-}$ ions across the nanoarchitectured film. The electrochemical characterization conclusively reveals that the magnitude of the voltammetric signal, which reflects the transport of $\mathrm{Fe}(\mathrm{CN})_{6}^{4-/ 3-}$ ions towards the underlying conductive support, is not affected by the switching from below to above LCST. Interestingly, the only electrochemical feature that changes upon increasing temperature is the redox potential of $\mathrm{Fe}(\mathrm{CN})_{6}{ }^{4-3-}$ ions which displays a marked cathodic shift upon increasing temperature above LCST. This can be explained by considering previous work by Anson and co-workers. ${ }^{49}$ These authors demonstrated that the interaction of $\mathrm{Fe}(\mathrm{CN})_{6}{ }^{4-/ 3-}$ with polyelectrolytes bearing quaternary amines promote a strong cathodic shift in the redox potential of the electroactive ions. As a consequence, we hypothesize that upon increasing temperature above the LCST, the collapse of the microgel domains within the multilayer leads to the exposure of PDACMAC layers to the incoming $\mathrm{Fe}(\mathrm{CN})_{6}^{4-/ 3-}$ ions, this being reflected by the shift in the redox potential.

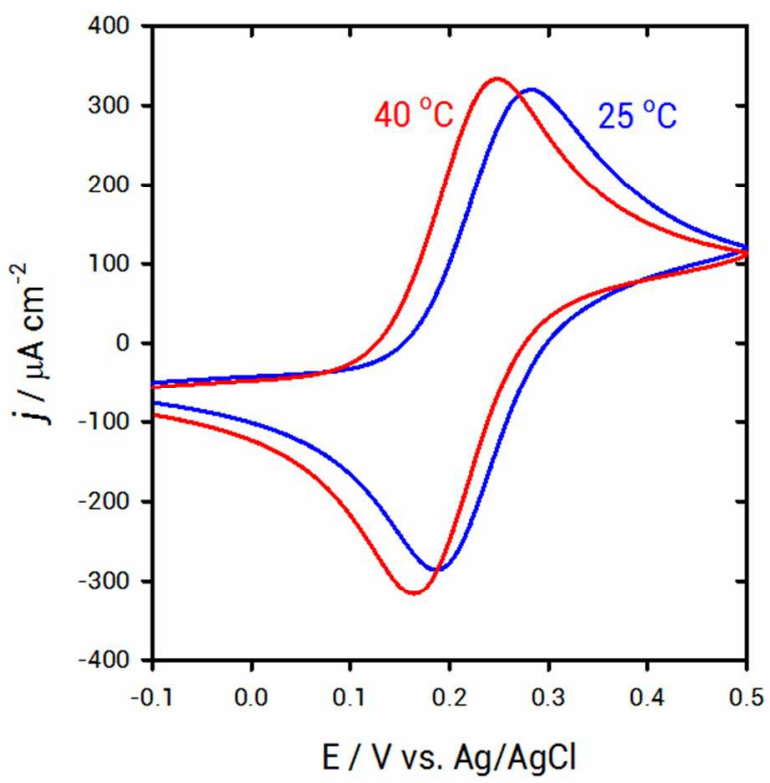

Figure 6. Voltammograms of (PNIPAM-co-PMAA/PDADMAC) ${ }_{9} /$ PNIPAM-co-PMAA multilayers performed at $25{ }^{\circ} \mathrm{C}$ (blue) and $40{ }^{\circ} \mathrm{C}$ (red), in contact with electrolyte solutions containing $\left[\mathrm{Fe}(\mathrm{CN})_{6}\right]^{-4 / 3} \cdot v=0.050 \mathrm{Vs}^{-1}$. 
Taken all together, the set of experiments can be interpreted considering that the opal-like microgel multilayer act as thermoresponsive matrix containing arrays of three-dimensional interconnected pores. Upon switching the temperature from below to above the LCST, films exhibit an increase in hydrophobicity, stiffness and adhesion properties. However, due the hierarchically organized, nanoarchitectured nature of the film, the switch in hydrophilicity and nanomechanical properties undergone by the self-assembled microgels does not compromise the porosity of the film, thus facilitating the free diffusion of redox probes through the polymer-free volume of the submicrometer pores generated in the inter-microgel cavities.

To the best of our knowledge, this is the first experimental report on layer-by-layer assembled films combining conflicting properties, such as stiffness and permeability. Our experimental results demonstrate that by drawing upon a "nanoarchitectonic approach" it is possible to decouple strongly correlated properties in order to create platforms that can switch stiffness, adhesion and wettability without affecting their high permeability properties.

\section{Conclusions}

We have demonstrated that by integrating organized microgel domains in polyelectrolyte multilayers we were able to decouple divergent, but strongly correlated functional properties in thin film architectures. In particular, we have shown that the layer-by-layer construction of nanoarchitectured, opal-like thermoresponsive films offer an interesting interfacial design in which the archetypical switching in hydrophobicity, stiffness and adhesion properties displayed by PNIPAM-based films can be implemented without affecting or disrupting the high permeability of the nanostructured thermoresponsive layer. We interpreted the results and concluded that the unique properties ascribed to these films arise from the hierarchical structure of the microgel multilayer that facilitates the coexistence of conflicting events and mechanisms operating at macroscopic, nanoscopic and/or molecular scales. In other words, the nanoarchitectured nature of the film displaying spatiallyaddressed 3D domains is the key to manipulate functions that are predominant at different structural dimensions, and consequently to harmonize conflicting properties. We consider that this conceptual framework based on "interfacial nanoarchitectonics" opens a new dimension: the possibility to create phase separated regions (functional domains) and concomitantly controlling their collective properties in order to decouple conflicting functions operating at different length scales.

This work offers a general concept that can be extended to other conflicting functions, thus opening new pathways to the fabrication of ultrathin multilayers displaying advanced concerted functions for potential use in a range of applications. The latter is particularly relevant if we consider that the use of microgels in LbL assembly retains the inherent simplicity of traditional LbL assembly, yet extends the range of nano- and mesoscale structural control that can be imparted to the interfacial architecture.

\section{Acknowledgements}

The authors acknowledge financial support from CONICET (PIP0370), and ANPCyT (PICT-20130905, PICT-2014-3792, PICT-2016-1680).

\section{References}

(1) McKee, J. R.; Appel, E. A.; Seitsonen, J.; Kontturi, E.; Scherman, O. A.; Ikkala, O. Healable, 
Stable and Stiff Hydrogels: Combining Conflicting Properties Using Dynamic and Selective Three-Component Recognition with Reinforcing Cellulose Nanorods. Adv. Funct. Mater. 2014, 24 (18), 2706-2713.

(2) Watanabe, H.; Vendamme, R.; Kunitake, T. Development of Fabrication of Giant Nanomembranes. Bull. Chem. Soc. Jpn. 2007, 80 (3), 433-440.

(3) Gwag, J. S.; Kim, Y.-K.; Lee, Y.-J.; Baek, J.-H.; Kim, J.-H. Anchoring Competition on Nanosurface Boundaries with Conflicting Mixed Nematic Anchoring Properties. Jpn. J. Appl. Phys. 2012, 51, 34102.

(4) Kamperman, M.; Synytska, A. Switchable Adhesion by Chemical Functionality and Topography. J. Mater. Chem. 2012, 22 (37), 19390-19401.

(5) Synytska, A.; Svetushkina, E.; Puretskiy, N.; Stoychev, G.; Berger, S.; Ionov, L.; Bellmann, C.; Eichhorn, K.-J.; Stamm, M. Biocompatible Polymeric Materials with Switchable Adhesion Properties. Soft Matter 2010, 6 (23), 5907-5914.

(6) Hammer, D. A.; Tirrell, M. Biological Adhesion at Interfaces. Annu. Rev. Mater. Sci. 1996, 26, 651-691.

(7) Mager, M. D.; Lapointe, V.; Stevens, M. M. Exploring and Exploiting Chemistry at the Cell Surface. Nat. Chem. 2011, 3 (8), 582-589.

(8) Skorb, E. V.; Andreeva, D. V. Surface Nanoarchitecture for Bio-Applications: Self-Regulating Intelligent Interfaces. Adv. Funct. Mater. 2013, 23 (36), 4483-4506.

(9) Sniadecki, N. J.; Desai, R. A.; Ruiz, S. A.; Chen, C. S. Nanotechnology for Cell-Substrate Interactions. Ann. Biomed. Eng. 2006, 34 (1), 59-74.

(10) Tang, Z.; Wang, Y.; Podsiadlo, P.; Kotov, N. A. Biomedical Applications of Layer-by-Layer Assembly: From Biomimetics to Tissue Engineering. Adv. Mater. 2006, 18 (24), 3203-3224.

(11) Yamato, M.; Akiyama, Y.; Kobayashi, J.; Yang, J.; Kikuchi, A.; Okano, T. TemperatureResponsive Cell Culture Surfaces for Regenerative Medicine with Cell Sheet Engineering. Prog. Polym. Sci. 2007, 32 (8-9), 1123-1133.

(12) Guest, J. K.; Prévost, J. H. Optimizing Multifunctional Materials: Design of Microstructures for Maximized Stiffness and Fluid Permeability. Int. J. Solids Struct. 2006, 43 (22-23), 7028-7047.

(13) Jaber, J. A.; Schlenoff, J. B. Polyelectrolyte Multilayers with Reversible Thermal Responsivity. Macromolecules 2005, 38 (4), 1300-1306.

(14) Lehaf, A. M.; Moussallem, M. D.; Schlenoff, J. B. Correlating the Compliance and Permeability of Photo-Cross-Linked Polyelectrolyte Multilayers. Langmuir 2011, 27 (8), 4756-4763.

(15) García, T. A.; Gervasi, C. A.; Rodríguez Presa, M. J.; Otamendi, J. I.; Moya, S. E.; Azzaroni, O. Molecular Transport in Thin Thermoresponsive poly(N -Isopropylacrylamide) Brushes with Varying Grafting Density. J. Phys. Chem. C 2012, 116 (26), 13944-13953.

(16) Song, S.; Hu, N. "On-Off” switchable Bioelectrocatalysis Synergistically Controlled by Temperature and Sodium Sulfate Concentration Based on poly(N -Isopropylacrylamide) Films. J. Phys. Chem. B 2010, 114 (17), 5940-5945.

(17) Prokopović, V. Z.; Duschl, C.; Volodkin, D. V. Hyaluronic Acid/poly-L-Lysine Multilayers Coated with Gold Nanoparticles: Cellular Response and Permeability Study. Polym. Adv. Technol. 2014, 25 (11), 1342-1348.

(18) Chen, Y.; Zhou, S.; Li, Q. Computational Design for Multifunctional Microstructural Composites. Int. J. Mod. Phys. B 2009, 23 (06n07), 1345-1351. 
(19) Ariga, K.; Li, J. Nanoarchitectonics for Advanced Materials: Strategy beyond Nanotechnology. Adv. Mater. 2016, 28 (6), 987-988.

(20) Aono, M.; Bando, Y.; Ariga, K. Nanoarchitectonics: Pioneering a New Paradigm for Nanotechnology in Materials Development. Adv. Mater. 2012, 24 (2), 150-151.

(21) Ariga, K.; Malgras, V.; Ji, Q.; Zakaria, M. B.; Yamauchi, Y. Coordination Nanoarchitectonics at Interfaces between Supramolecular and Materials Chemistry. Coord. Chem. Rev. 2016, 320-321, $139-152$.

(22) Ariga, K.; Ji, Q.; Hill, J. P.; Bando, Y.; Aono, M. Forming Nanomaterials as Layered Functional Structures toward Materials Nanoarchitectonics. NPG Asia Mater. 2012, 4 (5), e17-11.

(23) Serpe, M. J.; Jones, C. D.; Lyon, L. A. Layer-by-Layer Deposition of Thermoresponsive Microgel Thin Films. Langmuir 2003, 19 (21), 8759-8764.

(24) Nolan, C. M.; Serpe, M. J.; Lyon, L. A. Thermally Modulated Insulin Release from Microgel Thin Films. Biomacromolecules 2004, 5 (5), 1940-1946.

(25) Serpe, M. J.; Lyon, L. A. Optical and Acoustic Studies of pH-Dependent Swelling in Microgel Thin Films. Chem. Mater. 2004, 16 (22), 4373-4380.

(26) Clarke, K. C.; Lyon, L. A. Modulation of the Deswelling Temperature of Thermoresponsive Microgel Films. Langmuir 2013, 29 (41), 12852-12857.

(27) Zhang, L.; Spears, M. W.; Lyon, L. A. Tunable Swelling and Rolling of Microgel Membranes. Langmuir 2014, 30 (26), 7628-7634.

(28) Höfl, S.; Zitzler, L.; Hellweg, T.; Herminghaus, S.; Mugele, F. Volume Phase Transition of "smart" Microgels in Bulk Solution and Adsorbed at an Interface: A Combined AFM, Dynamic Light, and Small Angle Neutron Scattering Study. Polymer (Guildf). 2007, 48 (1), 245-254.

(29) Schmidt, S.; Motschmann, H.; Hellweg, T.; von Klitzing, R. Thermoresponsive Surfaces by Spin-Coating of PNIPAM-Co-PAA Microgels: A Combined AFM and Ellipsometry Study. Polymer (Guildf). 2008, 49 (3), 749-756.

(30) Decher, G.; Hong, J.-D. Buildup of Ultrathin Multilayer Films by a Self-Assembly Process, 1 Consecutive Adsorption of Anionic and Cationic Bipolar Amphiphiles on Charged Surfaces. Makromol. Chemie. Macromol. Symp. 1991, 46 (1), 321-327.

(31) Decher, G.; Hong, J. D.; Schmitt, J. Buildup of Ultrathin Multilayer Films by a Self-Assembly Process: III. Consecutively Alternating Adsorption of Anionic and Cationic Polyelectrolytes on Charged Surfaces. Thin Solid Films 1992, 210-211 (PART 2), 831-835.

(32) Schmidt, S.; Hellweg, T.; Klitzing, R. Von. Packing Density Control in P ( NIPAM-Co-AAc ) Microgel Monolayers : Effect of Surface Charge, $\mathrm{pH}$, and Preparation Technique. Langmuir 2008, 109 (7), 12595-12602.

(33) Nerapusri, V.; Keddie, J. L.; Vincent, B.; Bushnak, I. A. Swelling and Deswelling of Adsorbed Microgel Monolayers Triggered by Changes in Temperature, $\mathrm{pH}$, and Electrolyte Concentration. Langmuir 2006, 22 (11), 5036-5041.

(34) Chyasnavichyus, M.; Young, S. L.; Tsukruk, V. V. Mapping Micromechanical Properties of Soft Polymer Contact Lenses. Polymer (Guildf). 2014, 55 (23), 6091-6101.

(35) Giussi, J. M.; Velasco, M. I.; Longo, G. S.; Acosta, R. H.; Azzaroni, O. Unusual TemperatureInduced Swelling of Ionizable poly(N-Isopropylacrylamide)-Based Microgels: Experimental and Theoretical Insights into Its Molecular Origin. Soft Matter 2015, 11 (45), 8879-8886.

(36) Schmidt, S.; Zeiser, M.; Hellweg, T.; Duschl, C.; Fery, A.; Möhwald, H. Adhesion and 
Mechanical Properties of PNIPAM Microgel Films and Their Potential Use as Switchable Cell Culture Substrates. Adv. Funct. Mater. 2010, 20 (19), 3235-3243.

(37) Vanlandingham, M. R.; McKnight, S. H.; Palmese, G. R.; Elings, J. R.; Huang, X.; Bogetti, T. a.; Eduljee, R. F.; Gillespie, J. W. Nanoscale Indentation of Polymer Systems Using the Atomic Force Microscope. J. Adhes. 1997, 64 (1-4), 31-59.

(38) Overney, R. M. Nanotribological Studies on Polymers. Trends Polym. Sci. 1995, 3 (11), 359364.

(39) Shulha, H.; Kovalev, A.; Myshkin, N.; Tsukruk, V. V. Some Aspects of AFM Nanomechanical Probing of Surface Polymer Films. Eur. Polym. J. 2004, 40 (5), 949-956.

(40) LeMieux, M. C.; Lin, Y. H.; Cuong, P. D.; Ahn, H. S.; Zubarev, E. R.; Tsukruk, V. V. Microtribological and Nanomechanical Properties of Switchable Y-Shaped Amphiphilic Polymer Brushes. Adv. Funct. Mater. 2005, 15 (9), 1529-1540.

(41) LeMieux, M. C.; Peleshanko, S.; Anderson, K. D.; Tsukruk, V. V. Adaptive Nanomechanical Response of Stratified Polymer Brush Structures. Langmuir 2007, 23 (1), 265-273.

(42) Ye, C.; Drachuk, I.; Calabrese, R.; Dai, H.; Kaplan, D. L.; Tsukruk, V. V. Permeability and Micromechanical Properties of Silk Ionomer Microcapsules. Langmuir 2012, 28 (33), 1223512244.

(43) Chizhik, S. A.; Huang, Z.; Gorbunov, V. V.; Myshkin, N. K.; Tsukruk, V. V. Micromechanical Properties of Elastic Polymeric Materials as Probed by Scanning Force Microscopy. Am. Chem. Soc. Polym. Prepr. Div. Polym. Chem. 1998, 39 (2), 1146-1147.

(44) FitzGerald, P. A.; Dupin, D.; Armes, S. P.; Wanless, E. J. In Situ Observations of Adsorbed Microgel Particles. Soft Matter 2007, 3 (5), 580-586.

(45) Woodward, N. C.; Snowden, M. J.; Chowdhry, B. Z.; Jenkins, P.; Larson, I. Measurement of the Interaction Forces between Poly( N -Isopropylacrylamide-acrylic Acid) Microgel and Silica Surfaces by Colloid Probe Microscopy. Langmuir 2002, 18 (15), 2089-2095.

(46) Cole, M. A.; Voelcker, N. H.; Thissen, H.; Horn, R. G.; Griesser, H. J. Colloid Probe AFM Study of Thermal Collapse and Protein Interactions of poly(N-Isopropylacrylamide) Coatings. Soft Matter 2010, 6 (12), 2657-2667.

(47) Kidoaki, S.; Ohya, S.; Nakayama, Y.; Matsuda, T. Thermoresponsive Structural Change of a poly(N-Isopropylacrylamide) Graft Layer Measured with an Atomic Force Microscope. Langmuir 2001, 17 (8), 2402-2407.

(48) Cho, E. C.; Yong, D. K.; Cho, K. Temperature-Dependent Intermolecular Force Measurement of poly(N-Isopropylacrylamide) Grafted Surface with Protein. J. Colloid Interface Sci. 2005, 286 (2), 479-486.

(49) Kobayashi, J.; Anson, F. C. Association of Electroactive Counterions with Polyelectrolytes. 2. Comparison of Electrostatic and Coordinative Bonding to a Mixed Polycation-Polypyridine. $J$. Phys. Chem. 1991, 95 (6), 2595-2601. 


\section{Table of Contents Graphic}

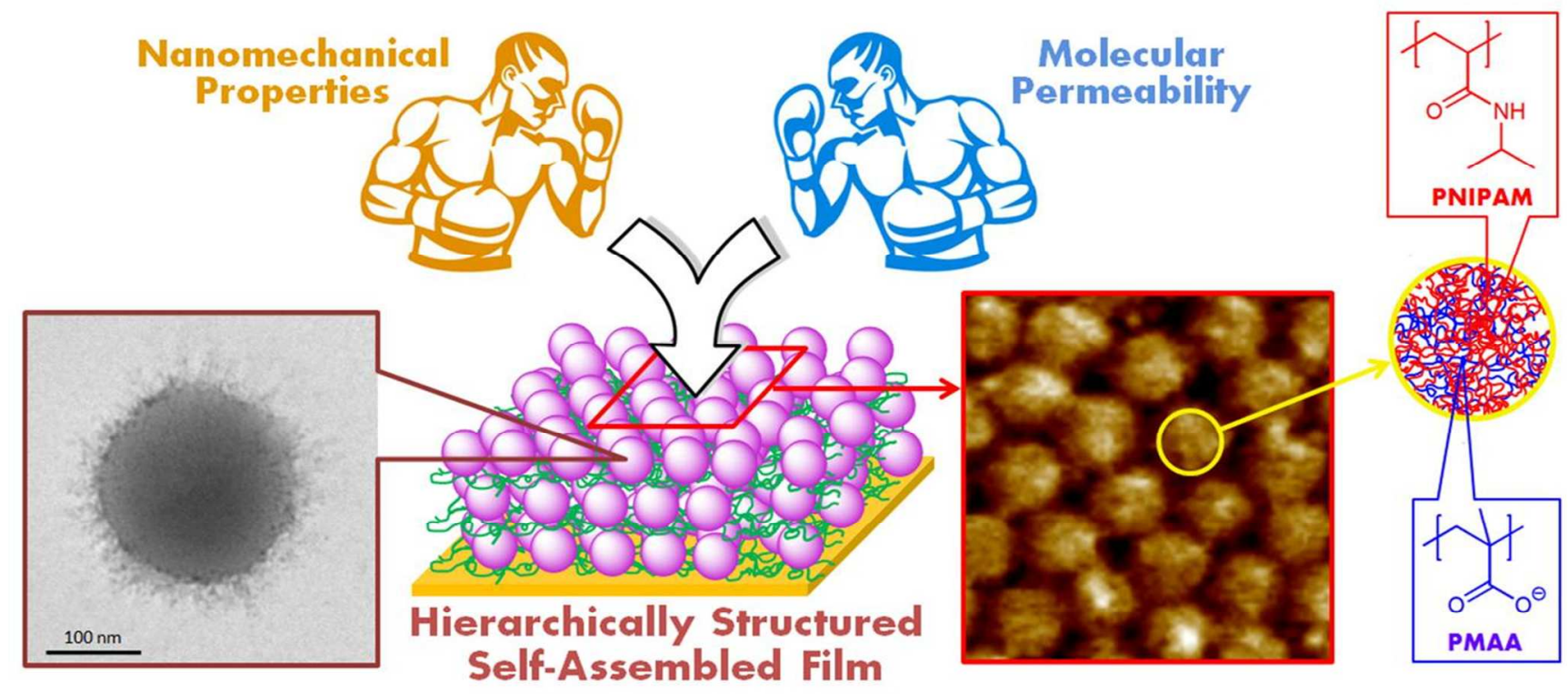

